\title{
ОСОБЕННОСТИ ДИАГНОСТИКИ И ХИРУРГИЧЕСКОГО ЛЕЧЕНИЯ БОЛЬШИХ И ГИГАНТСКИХ ФЕОХРОМОЦИТОМ
}

\author{
О. Н. Садриев ${ }^{1,2}$, А. Д. Гаибов ${ }^{1,2}$, Е. Л. Калмыков ${ }^{2}$, М. М. Маризоева ${ }^{3}$, А. Р. Кодиров ${ }^{4}$,
} О. Б. Бобджонова

${ }^{1}$ Республиканский научный центр сердечно-сосудистой хирургии, г. Душанбе, Республика Таджикистан,

${ }^{2}$ Таджикский государственный медицинский университет им. Абуали ибни Сино,

${ }^{3}$ Городской родильный дом № 2 Управления здравоохранения Хукумата, г. Душанбе,

${ }^{4}$ Городской медицинский центр Управления здравоохранения Хукумата, г. Душанбе

\section{PECULIARITIES OF DIAGNOSIS AND SURGICAL TREATMENT OF LARGE AND GIANT PHEOCHROMOCYTOMAS}

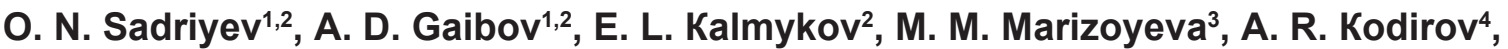 O. B. Bobjonova ${ }^{2}$}

${ }^{1}$ Republican Scientific Centre of Cardio-Vascular Surgery, Dushanbe, Republic of Tajikistan,

${ }^{2}$ Tajik State Medical University named after Abuali ibni Sino,

${ }^{3}$ Municipal Maternity Hospital № 2 of Health Department Khukumata, Dushanbe,

${ }^{4}$ Municipal Medical Centre of Health Department Khukumata, Dushanbe

\begin{abstract}
Реферат
Проанализированы результаты комплексной диагностики, предоперационной подготовки и хирургического лечения 13 пациентов по поводу больших и гигантских фреохромоцитом (ФХЦ). Всем больным проведен комплекс лабораторно-инструментальных методов исследования, включавший определение экскреции метанефринов (МН), ультразвуковое исследование (УЗИ), трехфазную компьютерную томографию (КТ), селективную рентгеноконтрастную ангиографию,что позволило правильно установить диагноз и определить тактику лечения. Несмотря на длительное существование артериальной гипертензии (АГ) и наличие типичных клинических проявлений ФХЦ, только у 2 пациентов диагноз установлен своевременно. Основным компонентом предоперационной подготовки явилось применение селективного а-адреноблокатора доксазозина в начальной дозе 4 мг с постепенным увеличением до 12 мг в сутки. Оперативное вмешательство выполнено у всех пациентов - расширенная адреналэктомия с удалением прилегающих участков жировой ткани и лимфатических узлов. Размеры удаленной ФХЦ составляли в среднем $(103,2 \pm 10,1) \times(93,1 \pm 8,2)$ мм, масса опухоли $(570,2 \pm 25,1)$ г. В ближайшем послеоперационном периоде у одного больного возникла серома операционной раны, у 2 - острая надпочечниковая недостаточность, которые успешно устранены консервативным путем. У всех больных ФХЦ соответствовала 4 - 7 баллам (по шкале PASS). В отдаленном периоде рецидивов заболевания не было. В ближайшем и отдаленном периоде две пациентки забеременели и родили живых доношенных детей.

Ключевые слова: феохромоцитома; $\alpha$-адреноблокаторы; адреналэктомия.

Abstract

Results of complex diagnosis, preoperative preparation and surgical treatment of 13 patients for large and giant pheochromocytomas are analyzed. To all the patients complex of laboratory-instrumental methods of investigation, including determination of the metanephrins excretion, ultrasonographic investigation, three-phase CT, selective roentgenocontrasting angiography was applied, what permitted to establish the diagnosis correctly and to determine the treatment tactics. In spite of a long-term existence of arterial hypertension and presence of typical clinical signs of pheochromocytomas, in 2 patients only the diagnosis was established timely. Application of selective a-adrenoblocking agent doxasosine in initial dose of $4 \mathrm{mg}$ with staged enhancement up to $12 \mathrm{mg}$ in a day was the main component of preoperative preparation. Operative intervention was performed in all patients - extended adrenalectomy with excision of adjacent parts of the fat tissue and lymphatic nodes. Dimensions of the excised pheochromocytomas have constituted (103.2 \pm 10.1$) \times(93.1 \pm 8.2)$ $\mathrm{mm}$ at average, and tumoral mass - $(570.2 \pm 25.1) \mathrm{g}$. In immediate postoperative period in one patient postoperative wound seroma have occurred, in 2 - an acute suprarenal insufficiency, which were eliminated by applying of conservative method. In all the patients pheochromocytomas were characterized and have constituted $4-7$ points (in accordance to the PASS scale). In remote period the disease recurrence was absent. In immediate and remote period two womn-patients have got pregnant and gave a birth to living fullterm new-born children.

Keywords: pheochromocytoma; $\alpha$-adrenoblocking agents; adrenalectomy.
\end{abstract}

Внедрение высокоинформативных методов исследования, в частности, КТ и магниторезонансной томографии (МРТ) позволило с высокой точностью диагностировать различные опухоли надпочечников, в том числе ФХЦ, на ранних стадиях, до появления глубоких гормональных изменений и нарушения функций жизненно важных органов [1, 2]. Однако сегодня частота выявления больших ФХЦ составляет от 1,2 до 29,6\% [2 - 5]. Причинами поздней диагностики ФХЦ в основном явля- ются неспецифичность клинических проявлений, медленный рост опухоли, ее доброкачественный характер и небольшая частота, хотя одним из основных кинических признаков заболевания у большинства пациентов является тяжелый 
гипертензивный синдром [3, 6]. Рост ФХЦ составляет приблизительно от 0,3 до 11 мм в год, что соответствует увеличению риска малигнизации опухоли, распада и кровотечения в несколько раз [2, 5].

Наиболее тяжелым осложнением больших опухолей является прорастание в соседние анатомические образования и сращение с крупными сосудами, что сопровождается формированием отечного и/или ишемического синдромов и значительно усложняет техническое выполнение оперативного вмешательства $[3,7]$.

По поводу малых и средних ФХЦ адреналэктомию выполняют с применением видеоэндоскопических технологий, имеющих преимущества по сравнению с открытым доступом. Однако возможности осуществления эндоскопической адреналэктомии при лечении больших ФХЦ ограничены из-за трудности визуализации центральной вены опухоли, необходимости достаточной мобилизации опухоли, близкого расположения сосудов, что увеличивает риск возникновения кровотечения и тяжелых гемодинамических нарушений [3]. Выполнение открытой адреналэктомии позволяет более радикально, за более короткое время и с меньшим риском тяжелого ятрогенного повреждения выполнить оперативное вмешательство [3, $5,7]$.

Цель исследования: обобщение собственного опыта хирургического лечения пациентов по поводу больших и гигантских ФХЦ.

\section{МАТЕРИАЛЫ И МЕТОДЫ ИССЛЕДОВАНИЯ}

Проанализированы результаты комплексной диагностики и хирургического лечения 13 пациентов по поводу ФХЦ диаметром более 8 см в период с 2006 по 2016 г. Женщин было 9 (69,2\%), мужчин - 4 (30,8\%). Возраст больных от 17 до 58 лет, в среднем $(44,1 \pm 5,6)$ года. У 12 (92,3\%) пациентов отмечена односторонняя локализация (правосторонняя у 7, левосторонняя - у 5) опухоли, у 1 - двустороннее поражение надпочечников. Длительность заболевания от первых симптомов до госпитализации составила в среднем $(8,2$ $\pm 1,8)$ года.
Кроме тщательного анализа анамнеза заболевания и результатов ранее проведенных диагностических исследований, больным выполнены УЗИ, трехфазная спиральная КТ (СКТ), аортография и селективная артериография опухоли надпочечника.

У всех пациентов определяли экскрецию МН в суточной моче методом иммуноферментного анализа (ИФА) с использованием лабораторных реагентов MetCombi ELISA EIA4083 (Германия).

Применяли стандартные методы статистического анализа, определяли медиану (Ме), верхний (Q25) и нижний (Q75) квартили. Достоверность различий определяли с использованием критериев МаннаУитни, Краскела-Уоллиса. Различия показателей считали статистически значимыми при $\mathrm{p}<0,001$.

\section{РЕЗУЛЬТАТЫ}

Все пациенты госпитализированы для планового хирургического лечения по поводу ФХЦ в сроки в среднем через 5 лет после появления первых симптомов заболевания. При этом только 2 (15,4\%) пациентов периодически наблюдали эндокринолог и кардиолог, проводили консервативное лечение, остальных пациентов в течение длительного времени лечили по поводу гипертонической болезни.

Причинами позднего обращения больных явились ошибки в распознавании ФХЦ врачами первичного звена (у 11), длительное самостоятельное применение различных гипотензивных средств (у 5), самостоятельное лечение (у 4).

Клинические проявления больших и гигантских ФХЦ характеризовались значительной вариабельностью и непостоянными симптомами. Основным симптомом было повышение артериального давления (АД). Кризовое течение АГ (катехоламиновый криз) на фоне нормального АД отмечено у 10 (76,9\%) пациентов, на фоне исходно повышенного АД - у 3 (23,1\%).

У 8 (61,5\%) больных отмечены церебральные, кардиальные и офтальмологические осложнения длительной неконтролируемой АГ, в частности, постинфарктный кардиосклероз - у 1 (7,7\%), остаточные явления перенесенного геморрагического инсульта - у 1 (7,7\%), постинфарктный кардиосклероз и остаточные явления перенесенного геморрагического инсульта - у 1 (7,7\%), мерцательная аритмия - у 4 (30,7\%), частичное отслоение сетчатки - у 1 $(7,7 \%)$.

у 3 (23,1\%) женщин в анамнезе были сведения о неоднократном патологическом кровотечении из матки во время менструального цикла, у 1 пациентки после удаления ФХЦ правого надпочечника возникла беременность на фоне имеющейся с противоположной стороны ФХЦ [8].

Содержание МН в суточной моче составляло 1760 [1370; 3505] мкг/сут, в норме $6-115$ мкг/сут $(\mathrm{p}<0,001)$; норметанефрина - 1710 [1315; 3455] мкг/сут, в норме 10 - 146 мкг/сут (р $<0,001)$.

Основной целью применения лучевых методов диагностики была необходимость установления/исключения новообразования надпочечников как причины гипертензивного синдрома.

Проведение СКТ позволило изучить топографоанатомические взаимоотношения опухоли с соседними органами и анатомическими образованиями: при правосторонней локализации - с печенью, нижней полой и воротной венами, почкой; при левосторонней - с селезенкой, хвостом поджелудочной железы, левой почкой.

По данным УЗИ, новообразование располагалось в проекции надпочечников, было округлой или овальной формы, как правило, с ровными контурами, гомогенной или гетерогенной структуры, повышенной эхогенности, иногда отмечали анэхогенные участки, что соответствовало зонам деструкции. Ткань надпочечника не определялась. Размеры выявленных ФХЦ составили в среднем $(108,5 \pm 6,7)$ мм.

Основным методом визуализации ФХЦ в настоящее время является СКТ. ФХЦ была овальной, округлой или неправильной веретенообразной формы, гомогенной или гетерогенной структуры, показатели плотности От +28 до +42 ед. Н, средняя плотность без контрастирования $+26,1 \pm 4,2$ ед. Н. Диаметр выявленных ФХЦ по данным СКТ составил в среднем $(105,7 \pm 6,2)$ мм. 
При исследовании КТ-плотности в разных фазах выведения контрастного вещества ФХЦ отмечена тенденция к задержке контрастного вещества, плотность при контрастировании в среднем $+35,9 \pm 7,2$ ед. $\mathrm{H}$, при этом показатели плотности медленно снижались в течение от 11 до 14 мин, в среднем $(12,1 \pm 0,4)$ мин, что свидетельствовало о доброкачественном характере ФХЦ. Измерение этого показателя позволяло дифференцировать ФХЦ от других форм гормонально-активных опухолей надпочечников, в частности, адренокортикального рака, метастатической карциномы.

У больных в целях более тщательного изучения ангиоархитектоники опухоли, определения прорастания опухоли в крупные сосуды забрюшинного пространства, а также признаков их злокачественного перерождения пациентам проведена аортография с селективной артериографией сосудов надпочечника.

Необходимость ее проведения обоснована тем, что с помощью УЗИ и СКТ невозможно идентифицировать центральную надпочечниковую вену и артерии малого диаметра. Применение артериографии позволило более четко изучить особенности ангиоархитектоники опухоли, ее отношение к магистральным венам и артериям соседних органов, аорте и нижней полой вене. На основании комплексного применения топических методов диагностики выбирали хирургический доступ для удаления новообразования.

Основным компонентом предоперационной подготовки было применение $\alpha$-адреноблокатора пролонгированного действия доксазозина, который назначали в дозе 4 мг 2 раза в сутки внутрь, с постепенным повышением дозы до 12 мг/сут. В течение 5 - 7 сут пациентам для профилактики ортостатической гипотензии рекомендовали строгий постельный режим с одновременной коррекцией гиповолемии и гомеостаза. Длительность предоперационной подготовки в среднем (14,3 \pm 1,1) сут.

При госпитализации у всех пациентов отмечено повышение АД, при применении доксазозина - его достоверное снижение до нормы ( $<$ < 0,001).
Для достижения $\beta$-блокирующего эффекта при наличии тахикардии применяли кардиоселективные средства (метопролол, атенолол) после достижения $\alpha$-блокирующего эффекта доксазозина, через 5 - 7 сут.

В целях устранения мерцательной аритмии 4 пациентам дополнительно назначали дигоксин и калий-магниевую смесь (калия хлорид 4\% 30 мл, магния сульфат 2,4\% 10 мл, изотонический раствор натрия хлорида 500 мл).

Проведение комплексной патогенетически обоснованной предоперационной подготовки позволило у всех больных стабилизировать нарушения гемодинамики и относительно безопасно произвести адреналэктомию.

Все пациенты оперированы в плановом порядке с применением торакофренолюмботомного доступа, оптимального для выполнения адреналэктомии по поводу больших ФХЦ, обеспечивающего оптимальный угол операционного действия, что позволяет выполнить операцию радикально и безопасно [7].

Удаление больших опухолей правого надпочечника имеет некоторые особенности, в частности, зону впадения центральной вены опухоли и зону сосудистой ножки правой почки. Выполнение операции сопряжено с риском их ятрогенной травмы, в связи с чем опухоль следует мобилизовать из нижнего ее полюса и снизу от сосудистой ножки правой почки [2].

Из-за больших размеров новообразования лигирование центральной вены надпочечника на ранних этапах адреналэктомии неосуществимо, поскольку она отходит от оставшейся небольшой части ткани самой железы, расположенной высоко и в глубине, из-за смещения ее опухолью.

Определенные трудности при левосторонней адреналэктомии возникают при визуализации хвоста поджелудочной железы и мобилизации центральной вены опухоли, которая впадает в почечную вену. Во избежание травмы сосудистой ножки левой почки необходима ее деликатная мобилизация и взятие на сосудистые турникеты для адекватного контроля при необходимости. В наших наблюдениях у всех больных выполнена расширенная адреналэктомия. Размеры удаленных ФХЦ составили в среднем $(103,2 \pm 10,1) \times$ $(93,1 \pm 8,2)$ мм, масса - $(570,2 \pm 25,1)$ г.

После операции специфических осложнений не было. Все пациенты живы, в одном наблюдении возникла серома операционной раны. Дренажные трубки удаляли на 3 - 7-е сутки после операции. В интра- и послеоперационном периоде заместительная гормональная терапия применена у 2 пациентов.В раннем послеоперационном периоде нормализация АД отмечена у всех больных.

Гистологическая оценка удаленных ФХЦ произведена согласно шкале отсчета гистологических признаков, предполагающих малигнизацию опухоли (PASS) [9]. У всех больных ФХЦ соответствовали $4-7$ баллам.

В 3 наблюдениях ФХЦ имели альвеолярный вариант строения, в 2 - трабекулярный, в 8 - дискомплексированный.

По данным клинических, лабораторных и инструментальных методов топической диагностики рецидив заболевания не выявлен.

\section{ОБСУЖДЕНИЕ}

Проблема ранней диагностики ФХЦ является одной из актуальных в хирургии, что обусловлено относительно небольшой частотой, неспецифичностью клинических проявлений, сложностями диагностики в ранних стадиях заболевания [3, 6].

По данным литературы, частота выявления больших ФХЦ составляет 1,2 - 29,6\%, при этом риск возникновения сердечно-сосудистых осложнений увеличивается в несколько раз [2 - 5].

Гигантскими считают ФХЦ, диаметр которых превышает 10 см, мас$\mathrm{ca}-500$ г [3].

Жизнеугрожающими осложнениями ФХЦ считают острое нарушение мозгового и коронарного кровообращения, острую левожелудочковую недостаточность, расслоение стенки аорты, отек легких, разрыв опухоли с массивным кровотечением $[4,8]$.

Частота поздней диагностики ФХЦ составила 83,8\%, причинами этого является неспецифичность 
клинических проявлений заболевания [4]. Сходные данные получены и нами, 84,6\% пациентов обратились поздно вследствие неправильной диагностики и лечения.

По нашему мнению, показатели выживаемости этих пациентов обусловлены адаптацией сердечно-сосудистой системы к резким перепадам АД.

Вместе с тем, поздняя диагностика ФХЦ является основным фактором увеличения размеров опухоли, повышения риска малигнизации и таких деструктивных изменений в ней, как некроз, кальцификация, кровоизлияние.

Для получения максимальной информации об анатомо-топографических характеристиках больших новообразований, кроме применения УЗИ и СКТ, авторы рекомендуют проведение аортографии с селективной артериографией опухоли. По нашему мнению, такая тактика оправдана, селективная ангиография позволяет более четко изучить особенности ангиоархитектоники опухоли и ее взаимоотношения с магистральными сосудами забрюшинного пространства.

В последние годы при адреналэктомии все чаще используют эндовидеотехнологию и миниинвазивные доступы. При применении миниинвазивных доступов отмечают небольшую частоту интра- и послеоперационных осложнений, незначительно выраженный болевой синдром, уменьшение продолжительности лечения пациентов в стационаре, быстрое восстановление трудоспособности, хороший косметический эффект [3].

Однако, учитывая особенности кровоснабжения надпочечников при наличии больших новообразований, предпочтение отдают выполнению адреналэктомии с использованием открытых хирургических доступов, из которых оптимальным является торакофренолюмботомный $[3,7]$. Доступ травматичный, однако только широкое поле операционного действия позволяет хирургу радикально удалить большие опухоли надпочечников, при необходимости - осуществить диссекцию регионарных лимфатических узлов, избежать ятрогенной травмы сосудов и органов забрюшинного пространства, а также массивной кровопотери.

После операции, выполненной с применением больших доступов, возможно возникновение кровотечения, эмпиемы плевры, гемо- и пневмоторакса, послеоперационного панкреатита, различных гнойных осложнений заживления раны.

В ранние сроки после своевременно выполненного хирургического вмешательства по поводу ФХЦ нормализацию АД отмечают практически у всех пациентов, однако в средне-отдаленном периоде вследствие появления истинных или ложных рецидивов у 11,2\% пациентов возобновляются клинические проявления заболевания, повышается АД [10].

Частота истинного рецидива ФХЦ составляет 6,15\%, его причинами являются мультицентрический характер опухоли, распространение опухоли на окружающие органы и ткани, наличие сосудистых опухолевых эмболов $[2,10]$. Высокая частота возникновения рецидива отмечена при удалении опухоли с оставлением интактной части органа. Вместе с тем, в наших наблюдениях в сроки от 7 мес до 8 лет рецидива опухоли не было. Возможно, это обусловлено выполнением расширенной адреналэктомии с удалением интактных участков прилегающей жировой клетчатки.

\section{Выводы}

1. Комплексная диагностика, адекватная предоперационная подготовка и выполнение расширенной адреналэктомии по поводу больших и гигантских ФХЦ с применением торакофренолюмботомного доступа позволяют у большинства пациентов достичь хороших результатов.

2. Наличие цереброкардиальных и офтальмологических осложнений ФХЦ не является абсолютным противопоказанием к проведению оперативного лечения.

3. Во избежание ятрогенной травмы крупных сосудов забрюшинного пространства и радикального удаления опухоли адреналэктомию по поводу больших ФХЦ следует выполнять в специализированных клиниках, располагающих достаточным опытом оперативных вмешательств при опухолевом поражении надпочечников.

\section{REFERENCES}

1. Sadriev ON, Gaibov AD, Gulmuradov TG, Anvarova ShS. Vozmozhnosti luchevykh metodov issledovaniya $v$ diagnostike opukholey nadpochechnikov. Rossiyskiy mediko-biologicheskiy vestnik imeni akademika I. P. Pavlova. 2016;(2):107-16. [In Russian].

2. Gaibov AD, Anvarova ShS, Kamolov AN, i dr. Nekotorye osobennosti diagnostiki i lecheniya bolnykh s opukholyami nadpochechnikov. Vestnik Avitsenny. 2011;(4):19 - 24. [In Russian].

3. Bondarenko VO, Lutsevich OE. Topograficheskaya diagnostika i khirurgicheskie vmeshatelstva pri gigantskikh feokhromotsitomakh nadpochechnika. Khirurgiya. Zhurn. im. N. I. Pirogova. 2011;(3):13-8. [In Russian].

4. Romashchenko PN, Maystrenko NA, Pashchenko OB, i dr. «Nemye» feokhromotsitomy. Vestnik khirurgii im. I. I. Grekova. 2004;163(3):227. [In Russian].

5. Privalov YuV, Kulikov LK, Bykova NM, Sobotovich VF. Diagnostika i khirurgicheskoe lechenie feokhromotsitom u patsientov s intsidentalomami nadpochechnikov. Novosti khirurgii. 2013;21(5):24-30. [In Russian].
6. Sadriev ON, Gaibov AD, Kalmykov EL, Anvarova ShS. Kompleksnaya diagnostika i khirurgicheskaya taktika pri dvukhstoronnikh feokhromotsitomakh. Rossiyskiy mediko-biologicheskiy vestnik im. akademika I. P. Pavlova. 2016;24(3):118-25. [In Russian].

7. Sadriev ON, Gaibov AD. Obosnovanie vybora khirurgicheskogo dostupa dlya adrenalektomii. Novosti khirurgii. 2015;23(2):145-54. [In Russian].

8. Sadriev ON, Gaibov AD, Kalmykov EL. Feokhromotsitoma pri beremennosti. Annaly khirurgii. 2016;21(6):407-10. [In Russian].

9. Thompson LDR. Pheochromocytoma of the adrenal gland scaled score (PASS) to separate benign from malignant neoplasms: A clinicopathologic and Immunophenotypic study of 100 cases. Am J Surg Pathol. 2002;26(5):551-66. PMID: 11979086.

10. Beltsevich DG, Kuznetsov NS. Prichiny retsidivov u bolnykh s opukholyami khromaffinnoy tkani. Khirurgiya. Zhurn. im. N. I. Pirogova. 2002;(8):19-23. [In Russian]. 